\title{
Laking of blood by freezing and thawing.
}

\author{
By C. C. Guthrie and M. E. Lee (by invitation).
}

[From the Physiological Laboratory, University of Pittsburgh.]

The mechanism of laking by freezing and thawing ${ }^{1}$ is not understood. Since it is known that drying through evaporation will cause laking, it is possible that drying through crystallization of water ${ }^{2}$ may account for laking by freezing. But again since it is known that hypertonic solutions may cause laking, and since there is evidence that the freezing point of serum is somewhat higher than that of the intracellular liquids and therefore in freezing a concentration of the serum solids occurs, it may be that such laking is fundamentally the same as laking by hypertonic solutions.

It was observed that blood could be repeatedly frozen and thawed as in freezing point measurements with slight or no laking. But when blood was exposed a single time to a temperature considerably below the freezing point for some time, on thawing strong laking occurred. To gain information on the question as to what degree of cooling is necessary to cause laking and also as to the relation of time of exposure to such temperature to the degree of laking, the experiments, the results of which are herein reported, were performed. The blood of various animals including ox, $\mathrm{dog}$, cat, and fowl has been used. Thus far no marked differences in the behavior of the different bloods have been observed. Summarized, the results show:

I. That slight or no laking occurs when the temperature of the blood is sufficiently lowered for the formation of crystals of ice if the blood is maintained at this temperature for but a short time.

2. When maintained at a temperature between a point slightly lower than that of the freezing point and minus one degree centigrade for ten minutes or more, laking occurs after the tube has remained at room temperature for some time.

3. The degree of laking, within limits, is proportionate to the

${ }^{1}$ Rollett, Sitzgsber, d. Wiener Acad., XLVI, S, 65, 1862.

${ }^{2}$ Cf. Müller-Thurgan, Landw. Jahrb., XV, 534, 1886. 
degree to which the temperature was lowered, and to the length of time during which the low temperature was maintained.

\section{3 (9ro)}

\section{Laking of blood by drying.}

By C. C. GuTHRIE and M. E. LEe (by invitation).

\section{[From the Physiological Laboratory, University of Pittsburgh.]}

Drying of blood may cause laking of the scarlet blood discs. ${ }^{1}$ To obtain more information regarding the phenomenon, the expeiments reported in this communication were undertaken.

The phenomenon may be produced in a number of ways. Perhaps the simplest is to prepare an ordinary wet blood mount and to observe it with a microscope. Another method is to make an ordinary blood smear and allow it to dry quickly in the air and then place it film side down on a microscope slide and after focusing it under a microscope, place a drop of serum, salt solution or other liquid on the slide, so that the edge of the drop forms contact with the edge of the slip and will spread under it.

Under these conditions laking occurs and may be readily observed in the individual discs.

In the case of a wet mount, the process is slow and various stages may be seen. If drying be rapid the individual discs may lose their hemoglobin with slight or no change in size. But if it be more gradual, they may be seen to swell before laking. This is also the case with shrunken or crenated discs.

In the case of dry mounts, the hemoglobin is almost instantaneously dissolved on contact with the liquid under the slip. If any change in the size of the discs occurs, it has not been observed.

At present the manner in which laking is thus produced is not known. Drying is, of course, accompanied by concentration of salts or other substances present in solution or suspension both within the discs and in the serum. Now it is known that hypertonic solutions of such substances if caused to act on the blood may produce laking. Therefore, the question arises, Is laking

\footnotetext{
${ }^{1}$ Am. Jr. Phy., I903, VIII, 44I.
} 